\title{
Problèmes pratiques posés par la production du vide dans les systèmes d'attaques sous plasma et L.P.C.V.D. ( $\left.{ }^{*}\right)$
}

\author{
P. Duval \\ CIT-ALCATEL, 98, avenue de Brogny, 74000 Annecy, France
}

(Reçu le 3 juin 1980, accepté le 9 juin 1980)

\begin{abstract}
Résumé. - Le développement, à l'échelle industrielle, des nouveaux procédés de production des micro-circuits pose de nouveaux problèmes pour l'obtention du vide qui leur est nécessaire. Ces problèmes résultent des fluides corrosifs et dangereux mis en œuvre ainsi que des grandes quantités de particules abrasives que les pompes à vide doivent absorber. On examine les modifications qu'il faut faire subir aux systèmes de pompage, les nouveaux fluides de lubrification à utiliser, ainsi que les précautions à prendre pour assurer la sécurité des installations, et les impératifs de maintenance nécessités par cette nouvelle chimie sous vide.
\end{abstract}

\begin{abstract}
Semiconductor manufacturing trends have initiated industrial scale implementation of processes (L.P.C.V.D., L.T.O., Plasma Etching, Plasma Deposition, etc.) employing corrosive or dangerous gases. The operating range of these systems $(0.5$ to $100 \mathrm{~Pa})$ is bracketed by the application limits of oil-sealed vane or oscillating-piston vacuum pumps. Creating a vacuum in these new machines poses unprecendented problems for the designer, however. The development of vacuum pumps and their auxiliary equipment is traced, emphasizing the need for a good seal and the elimination under high-corrosion conditions of solid particles generated either in the process itself or by secondary reactions in the pump. Selection of correct lubricating oils for mechanical pumps ensures their proper function and protects operating personnel.
\end{abstract}

Les applications classiques du vide à l'industrie des semi-conducteurs (évaporation, sputtering), si elles nécessitent des pompes performantes, ne sont que peu contraignantes, car il s'agit de pomper des fluides inertes et propres. Cette situation est en train d'évoluer rapidement et assez radicalement, et l'on voit apparaitre des méthodes de production des circuits à semi-conducteur dans lesquelles il est fait appel à une " chimie sous vide " en remplacement des méthodes dites physiques. Ces méthodes, L.P.C.V.D. [14, 12, 15, 24] L.T.O. [20], Attaque sous plasma, (Plasma-Etching, R.I.E.) [18-36], C.V.D. Plasma [23, 29] et autres, ont en commun l'utilisation de gaz actifs, dangereux, et souvent corrosifs. La plupart sont des substances halogénées telles que :

- $\mathrm{CF}_{4}[19,11,5]$,

- $\mathrm{CCl}_{4}[24,31], \mathrm{SiF}_{4}[3], \mathrm{C}_{3} \mathrm{~F}_{8}$ [8], $\mathrm{C}_{2} \mathrm{~F}_{6}$ [11],

- $\mathrm{SiH}_{4}[14,35], \mathrm{SiH}_{2} \mathrm{Cl}_{2}[1,15,30]$,

- $\mathrm{SiCl}_{4}$ [15], etc...

(*) Conférence présentée aux Journées de Physique Industrielle de la S.F.P. à Toulouse (mars 1980).
Il faut ajouter à cela que l'implantation ionique met en œuvre des substances (arsine, phosphine...) qui ne sont guère plus sympathiques!

Tous ces corps sont introduits dans l'enceinte de dépôts, seuls ou mélangés à d'autres (oxygène par exemple en plasma-etching) et c'est de leur décomposition que résultent les effets recherchés. Les réactions sont complexes $[7,27,8]$ et les produits de décomposition que les pompes doivent évacuer sont nombreux : produits initiaux, acides $(\mathrm{HCl})$, radicaux halogénés libres $\left(\mathrm{F}^{+} \mathrm{Cl}^{+}\right)$, oxygène...

Il se pose donc maintenant, tant à l'utilisateur qu'au constructeur de pompes à vide, des problèmes nouveaux de :

- Sécurité pour le personnel.

- Corrosion des pompes et de leurs périphériques.

- Maintenance.

- Fiabilité.

Ces problèmes varient d'un procédé à l'autre [9], et nous avons tenté de les résumer dans le tableau I.

Une installation de dépôt, ou de traitements sous vide, n'est pas pompée par une pompe à vide, mais par un système de pompage plus ou moins complexe 
Tableau I. - Les principaux problèmes nouveaux pour le pompage en L.P.C.V.D. Attaque sous plasma. Implantation ionique.

\begin{tabular}{|c|c|c|c|}
\hline & $\begin{array}{c}\text { L.P.C.V.D. } \\
\text { L.T.O. } \\
\text { C.V.D. PLASMA }\end{array}$ & $\begin{array}{c}\text { ATTAQUE SOUS PLASMA } \\
\text { PLASMA ETCHING } \\
\text { R.I.E. }\end{array}$ & IMPLANTATION IONIQUE \\
\hline SECURITE & $\begin{array}{l}\text { Produits toxiques } \\
\text { Usage du silane et de } \\
\text { ses dérivés explosifs }\end{array}$ & $\begin{array}{l}\text { Produits toxiques } \\
\text { Oxygène }\end{array}$ & $\begin{array}{l}\text { Produits toxiques } \\
\text { Poisons (Arsine Phosphine) }\end{array}$ \\
\hline & \multicolumn{2}{|c|}{ USAGE DES PIEGES } & \\
\hline CORROSION & $\begin{array}{l}\text { Assez faible en } \\
\text { général } \\
\text { Surtout due au } \mathrm{HCl}\end{array}$ & Importante & $\begin{array}{l}\text { Moyenne } \\
\text { Concentrée au pompage de } \\
\text { la source }\end{array}$ \\
\hline MAINTENANCE & $\begin{array}{l}\text { Important problème de } \\
\text { poussières et } \\
\text { particules solides } \\
\text { Faible dégradation de } \\
\text { l'huile }\end{array}$ & $\begin{array}{l}\text { Dégradation très } \\
\text { importante de l'huile } \\
\text { Usure due aux mauvaises } \\
\text { qualités lubrifiantes } \\
\text { des huiles synthétiques }\end{array}$ & Dégradation de l'huile \\
\hline
\end{tabular}

selon le problème à traiter, et les critères que nous venons d'énoncer, s'appliquent, bien évidemment, à la totalité de ce système. A quoi sert en effet d'utiliser une pompe à vide inusable, anti-corrosion et parfaitement étanche, si elle est connectée à des filtres qui fuient au moyen de tubes en matériaux non appropriés ?C'est donc toute la ligne de pompage, de l'enceinte de dépôt au scrubber final, qui doit faire l'objet d'une construction de qualité.

1. La sécurité. - 1.1 Etanchéité. Sécurité. Beaucoup des gaz mentionnés ci-dessus sont toxiques, certains sont des poisons violents (arsine), d'autres sont susceptibles de constituer des mélanges détonnants avec l'air (silane, hydrogène), tous sont suffisamment dangereux pour qu'une attention particulière soit portée à l'étanchéité de tout le circuit de pompage, dans sa partie haute pression (refoulement), qui est généralement celle à laquelle on apporte le moins de soins. Une attention particulière doit être apportée au robinet de lest d'air lors du pompage de silane ou d'hydrogène et aux opérations de vidange et remplissage d'huile durant lesquelles il est malheureusement aisé de créer des fuites; il est donc recommandé de mécaniser ces opérations en installant des vannes d'arrêt sur les canalisations correspondantes. On pensera également à l'étanchéité de tous les circuits auxiliaires.

Il faut se souvenir qu'il s'agit autant d'empêcher les gaz de procédé de sortir dans l'atmosphère que d'interdire aux composants dangereux de celle-ci (oxygène et vapeur d'eau), de pénétrer dans le système de pompage.

1.2 LE POMPAGE DE L'OXYGÈNE. - L'attaque sous plasma (Plasma-etching, R.I.E.) utilise des mélanges

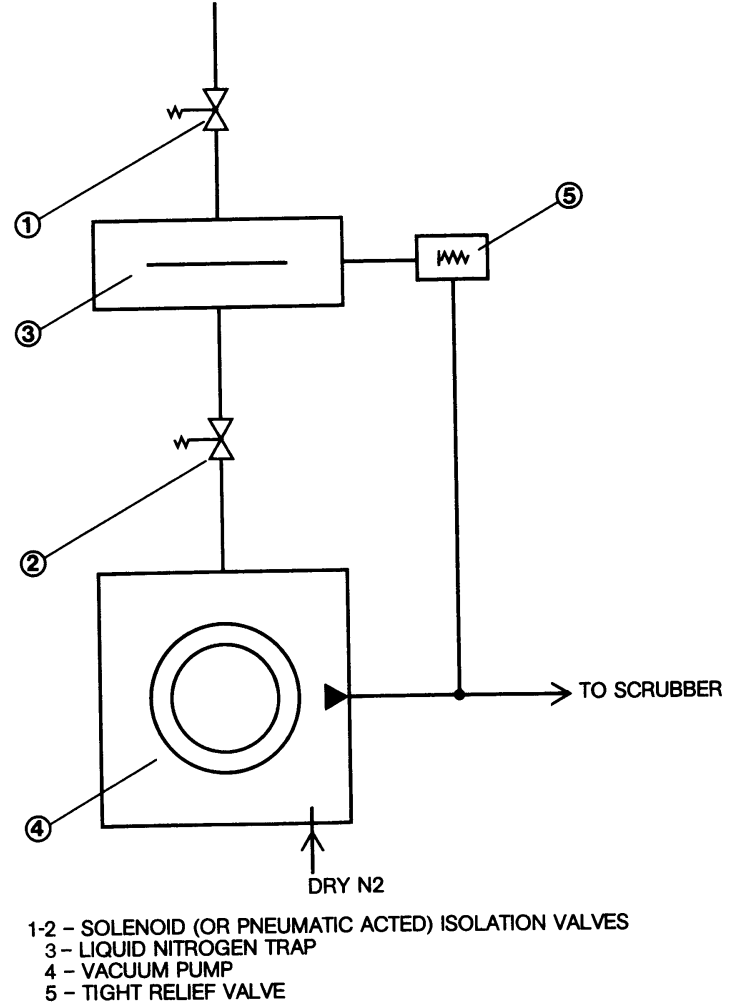

Fig. 1. - Montage normal d'un piège à azote liquide assurant la sécurité en cas de réchauffage.

[Monting a relief valve on $\mathrm{N} 21$ trap to prevent it against over pressure when accidental heating.]

contenant de l'oxygène à une concentration qui peut être élevée, et quelquefois de l'oxygène pur.

Il est indispensable d'avoir présent à l'esprit en permanence que les huiles d'origine minérale sont des combustibles. Ceci implique : 
a) qu'elles sont oxydées pendant le pompage et que, corrélativement, elles perdent rapidement leurs qualités lubrifiantes,

b) qu'il peut se constituer dans la pompe les conditions d'une explosion !

Il ne faut pas pour autant exagérer l'importance du problème, il y a tout de même $20 \%$ d'oxygène dans l'air que nous respirons ! Les précautions doivent donc être prises dès que l'on dépasse sensiblement cette concentration : certains utilisateurs mettent la frontière à $30 \%$ de concentration d'oxygène dans le mélange. En dessous de cette valeur, il est quand même conseillé de mettre en pratique les précautions décrites à propos de la corrosion (voir ci-dessous).

$\mathrm{Au}$-delà de cette limite, il faut proscrire l'usage d'huiles minérales et utiliser des fluides synthétiques tels que :

- fluides chlorocarbonés :

INLAND 41, HALOCARBON 100

ALCATEL 100

et surtout :

- perfluoroéther :

FOMBLIN L VAC 25-05, ALCATEL 113, DU

PONT KRYTOX 143 AY

(voir ci-après l'influence sur les caractéristiques).

Il est déconseillé d'utiliser des fluides tels que les Tri-Aryl phosphates esters (FYRQUEL 220) qui ont déjà provoqué de graves accidents [32].

1.3 UTILISATION DES PIÈGES. - On peut penser à protéger les pompes mécaniques en équipant leur circuit d'aspiration de pièges refroidis chargés de retenir les produits corrodants (ou indésirables) ou de piège à fixation à matériaux absorbants (charbon actif, tamis moléculaires,...) destinés au même usage. Il faut alors réfléchir sérieusement aux problèmes de sécurité que pose l'emploi de ces pièges. Il est nécessaire à ce sujet, de rappeler quelques caractéristiques des pièges :

a) un piège refroidi n'est efficace que tant qu'il est froid;

b) lors d'un réchauffement, qui peut être accidentel, comme lors d'un défaut d'alimentation en fluide frigorigène, les produits condensés s'évaporent et il peut en résulter une élévation considérable de la pression dans le circuit de pompage. Or la plupart des pièges à azote liquide ne sont pas munis de soupapes de sécurité et cette élévation de pression peut provoquer des fuites vers l'extérieur (voir plus haut)...,

c) le mélange de charbon actif, d'hydrocarbures, d'oxygène ou d'autres produits oxydants (radicaux fluorés par exemple) peut également conduire à des mélanges détonants;

d) dans un système utilisé à la fois pour faire des dépôts et de la gravure, on est conduit à mettre en œuvre successivement des gaz incompatibles entre eux (exemple silane puis oxygène). Il est alors dan- gereux d'en retenir un sur le piège qui ressemble un peu à un cocktail Molotov.

Des considérations de sécurité conduisent donc certains utilisateurs à proscrire l'usage de pièges dans leurs installations; le résultat est que les pompes à vide et leurs périphériques doivent avoir l'estomac solide!

2. La corrosion. - Comment se présente le problème de corrosion? Il faut d'abord bien voir que les gaz actifs sont introduits dans les pompes froids (car ils se sont refroidis dans les canalisations ou les pièges qui les précédent), et à une pression suffisamment basse, puisque inférieure à $100 \mathrm{~Pa}$ la plupart du temps, pour que les vitesses de corrosion soient modestes pour tous les organes internes des pompes fonctionnant sous vide. C'est le cas des rotors, des palettes et de l'intérieur des stators en particulier. Par contre, on constate que les produits corrodants s'accumulent dans l'huile de lubrification de la pompe ainsi que dans l'espace de refoulement à la pression atmosphérique, ce sera donc essentiellement les organes fonctionnant hors vide qui sont le plus sujéts à la corrosion puisque la concentration en agents corrodants y est la plus élevée. La corrosion concerne tous les composants des pompes à vide, aussi bien les matériaux solides, que les joints et l'huile dont le maintien des propriétés lubrifiantes est le point le plus important à prendre en considération pour maintenir constantes les caractéristiques des pompes à vide. La zone théoriquement la plus critique est l'espace de refoulement où les gaz sont fortement comprimés et donc chauds, ils sont expulsés au travers des soupapes de refoulement dont les matériaux élastomères sont très agressés. Un certain nombre d'acides, tel $\mathrm{HCl}$, sont relativement inertes à l'état anhydre mais deviennent hautement agressifs en présence d'eau. L'eau à l'état vapeur existe dans l'air atmosphérique et est souvent introduite accidentellement, ou involontairement dans les pompes. C'est la cause la plus fréquente de dégradation brutale par corrosion. Les précautions à prendre pour se protéger des méfaits de la vapeur d'eau sont simples :

- Veiller à la canalisation d'évacuation des effluents gazeux refoulés par les pompes à vide, en y introduisant un balayage de gaz neutre et sec (azote). Le mieux est de réaliser cette protection en injectant le gaz dans l'espace de refoulement de la pompe.

- Avoir un dispositif de lest d'air raccordé à de l'azote sec, et non à l'air atmosphérique.

- Vidanger et rincer les pompes avec de l'huile neuve non polluée avant un arrêt prolongé et les stocker sous protection d'azote. Un week-end prolongé est souvent meurtrier !

En ce qui concerne le choix des matériaux, il n'existe pas de solution miracle, il n'y a que des compromis moins mauvais que d'autres, car il n'existe pas de matériau résistant à toutes les formes de corro- 
sion. La meilleure protection contre la corrosion est de disposer de solutions adaptables aux différents problèmes rencontrés, et de maintenir dans la pompe de l'huile claire et propre.

3. L'huile. - La plupart des huiles pour pompes à vide poussé sont réactives avec les produits corrosifs et les résidus ainsi obtenus sont généralement des goudrons assez visqueux. Il s'ensuit que les différents orifices chargés de réguler l'introduction de l'huile dans la pompe peuvent être rapidement obturés. Par ailleurs, il faut se souvenir que les corps solides qui pénètrent dans la pompe (poussières submicroniques) finissent par se mélanger également à l'huile et le résultat donne quelquefois lieu à des surprises. Ainsi dans les installations de dépôts de silice par L.P.C.V.D., la silice absorbe l'huile et le mélange forme une crème qui fixe $\mathrm{HCl}$. Cette fixation accélère la dégradation de l'huile et le mélange est une sorte de gelée très thixotropique avec une tension superficielle élevée

$$
\mathrm{SiO}_{2}+\mathrm{HCl}+\text { huile } \rightarrow \text { gelée } .
$$

Cette gelée tend à se déposer partout dans la pompe et peut même obturer les tamis d'aspiration.

La seule solution applicable dans un tel cas, est d'utiliser des dispositifs de filtration d'huile et d'assurer un grand débit d'huile dans la pompe.

La question de la qualité de l'huile est très délicate, car elle ne peut être examinée que pour chaque cas particulier. On pourrait penser, a priori, qu'il suffit de choisir un fluide de lubrification inerte tel que les produits Fomblin [4] mais ce sont en fait les conditions économiques qui dictent le choix. Pourquoi en effet adopter une huile très coûteuse si on doit régulièrement démonter la pompe ou les accessoires qui lui sont associés, à cause des corps solides qu'il faut évacuer ? Par contre, la présence d'oxygène en attaque sous plasma, impose des fluides synthétiques. Le seul critère valable pour tous les fluides, outre ceux qui concernent leur viscosité ou leur pression de vapeur saturante, est leur pureté; il faut absolument proscrire les huiles qui contiennent des additifs (inhibiteurs de corrosion, par exemple) et celles susceptibles de donner des produits de décomposition dangereux (voir ci-après).

Le tableau II résume brièvement les principaux fluides utilisés dans les pompes à vide qui équipent les installations de L.P.C.V.D. ou attaque sous plasma. Tous les fluides sont utilisables quel que soit le type de pompe utilisé (à palettes ou à piston oscillant) pour autant que l'on adopte une viscosité convenable.

Les systèmes d'attaque sous plasma, qui produisent très peu de particules solides, peuvent avantageusement être équipés de systèmes de filtration d'huile avec cartouches absorbantes (terres activées, charbon actif...) qui retiennent les acides (Fig. 4). Ces filtres à acide doivent avoir un faible volume pour l'utilisation de fluides coûteux (chlorocarbonés ou Fomblin) afin de minimiser le coût des huiles. Les mêmes dispositions sont applicables aux pompes qui équipent les sources d'ions des implanteurs d'ions. Dans cette application, on peut citer une pompe utilisée pendant environ 2 ans sans changement de l'huile Fomblin qui l'équipe.

Tableau II. - Principal oils for vacuum pumps in difficult applications.

\begin{tabular}{|c|c|c|c|c|}
\hline TYPE & BRAND & $\begin{array}{l}\text { RELATIVE } \\
\text { PRICE }\end{array}$ & $\begin{array}{l}\text { RECOMMENDED } \\
\text { USE }\end{array}$ & LIMTATIONS \\
\hline $\begin{array}{l}\text { Pure Parafin-base } \\
\text { mineral oil }\end{array}$ & $\begin{array}{l}\text { INLAND ** } 15 \\
\text { ALCATEL * } 100 \\
\text { ALCATEL * } 101\end{array}$ & 1 & $\begin{array}{l}\text { General } \\
\text { LPCVD use }\end{array}$ & $\begin{array}{l}\text { Risks explosion in the } \\
\text { presence of oxygen }\end{array}$ \\
\hline \multirow[t]{2}{*}{$\begin{array}{l}\text { Molecular distilled } \\
\text { mineral oil }\end{array}$} & $\begin{array}{l}\text { INLAND ** } 19 \\
\text { ALCATEL * } 103\end{array}$ & 2 & $\begin{array}{l}\text { General } \\
\text { LPCVD use }\end{array}$ & \multirow{2}{*}{$\begin{array}{l}\text { Risks explosion in the } \\
\text { presence of oxygen }\end{array}$} \\
\hline & 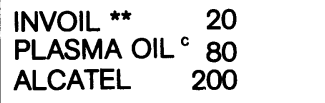 & 1,5 & $\begin{array}{l}\text { Low base pressure } \\
\text { Plasma etching } \\
\text { lon implant }\end{array}$ & \\
\hline \multicolumn{5}{|l|}{ SYNTHETIC FLUIDS } \\
\hline Chlorofluocarbon & $\begin{array}{l}\text { INLAND ** } 41 \\
\text { HALOCARBON }{ }^{n} 100 / 100 \\
\text { ALCATEL * } 110\end{array}$ & 75 & $\begin{array}{l}\text { Oxygen } \\
\text { pumping } \\
\text { Plasma etching }\end{array}$ & \\
\hline $\begin{array}{l}\text { Perfluorinated } \\
\text { polyether (PFPE) }\end{array}$ & $\begin{array}{l}\text { KRYTÓX d } \\
\text { ALCATEL } 113 \\
\text { FOMBLIN L VAC 25-5*** }\end{array}$ & 100 & $\begin{array}{l}\text { Plasma etching } \\
\text { lon implant } \\
\text { Oxygen pumping }\end{array}$ & \\
\hline $\begin{array}{l}\text { Triaryl Phosphate } \\
\text { ester }\end{array}$ & FYRQUEL ${ }^{s} 220$ & 3 & & $\begin{array}{l}\text { Potential hazard with } \\
\text { oxygen }\end{array}$ \\
\hline \multicolumn{3}{|c|}{ 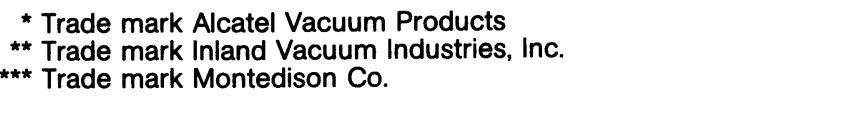 } & \multicolumn{2}{|c|}{$\begin{array}{l}\text { d Trade mark Dupont de Nemours Co. } \\
\text { n Trade mark Halocarbon Products Corp. } \\
\text { s Trade mark Stauffer Chemical Co. } \\
\text { c Trade mark C.V.C. Co. }\end{array}$} \\
\hline
\end{tabular}


3.1 INFLUENCE DE L'HUILE SUR LE VIDE LIMITE DES POMPES. - Toutes les huiles ne donnent pas le même résultat dans la même pompe; le paramètre qui est le plus affecté est la pression limite, qui dépend de la pression de vapeur saturante du fluide de lubrification, mais aussi de la viscosité et de son aptitude à dissoudre les gaz. En particulier, les huiles synthétiques conduisent à une augmentation sensible du vide limite des pompes à 2 étages. A titre d'exemple, on obtient les valeurs suivantes sur une pompe de $30 \mathrm{~m}^{3} \mathrm{~h}^{-1}$ (ALCATEL $2033 \mathrm{C}$ ).

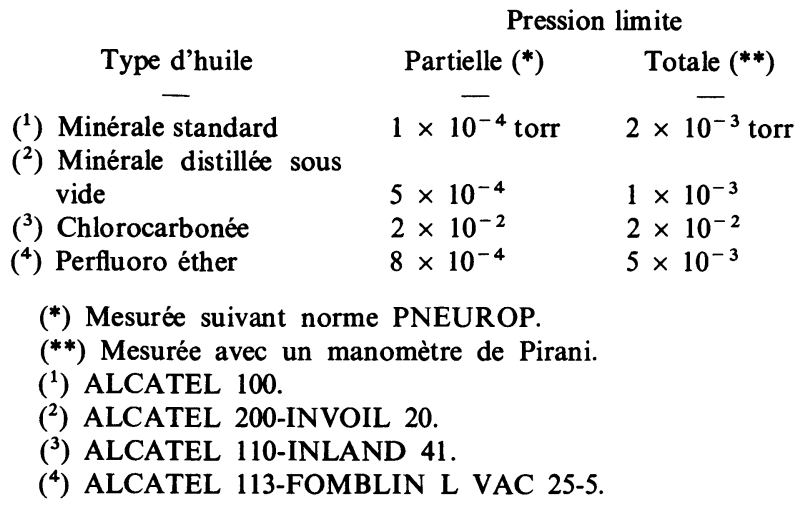

3. 2 LA PRESSION DE VAPEUR. - La pression de vapeur saturante d'une huile est importante, car elle donne, d'une part la pression limite que la pompe ne pourra pas dépasser, et d'autre part une bonne indication sur sa pureté dans le cas des huiles minérales. Une règle approximative (Thumb rule) peut s'énoncer comme suit " lors du choix d'une huile de pompe à vide devant fonctionner en présence de gaz corrosifs, il est bon de choisir un fluide dont la pression de vapeur (à température ambiante) est environ 100 fois plus basse que la pression limite de la pompe ".

La courbe, figure 2, donne les valeurs de pression de vapeurs des principales huiles pour pompes mécaniques.

3.3 LA LUBRIfICATION. - Il est impossible de qualifier par un seul critère mesurable, le pouvoir lubrifiant d'une huile, car il dépend de beaucoup de paramètres. Il faut tout de même retenir que les huiles synthétiques sont de très mauvais lubrifiants et que leur utilisation dans les pompes Roots n'est guère évidente. Les variations de viscosité en fonction $\mathrm{du}$ type d'huile sont indiquées sur la figure 3 .

4. Les particules solides. - Ce qui caractérise le L.P.C.V.D. et qui a fortement contribué à son développement est sa grande vitesse de dépôts ; le corollaire étant que la chambre de dépôt est une source importante de poussières. Certaines de ces poussières résultent du process, c'est le cas en particulier du chlorure d'ammonium dans la réaction qui conduit aux couches de nitrures de silicium $[14,15]$

$3 \mathrm{SiH}_{2} \mathrm{Cl}_{2}+7 \mathrm{NH}_{3} \rightarrow \mathrm{Si}_{3} \mathrm{~N}_{4}+6 \mathrm{NH}_{4} \mathrm{Cl}+\mathrm{NH}_{3}$.

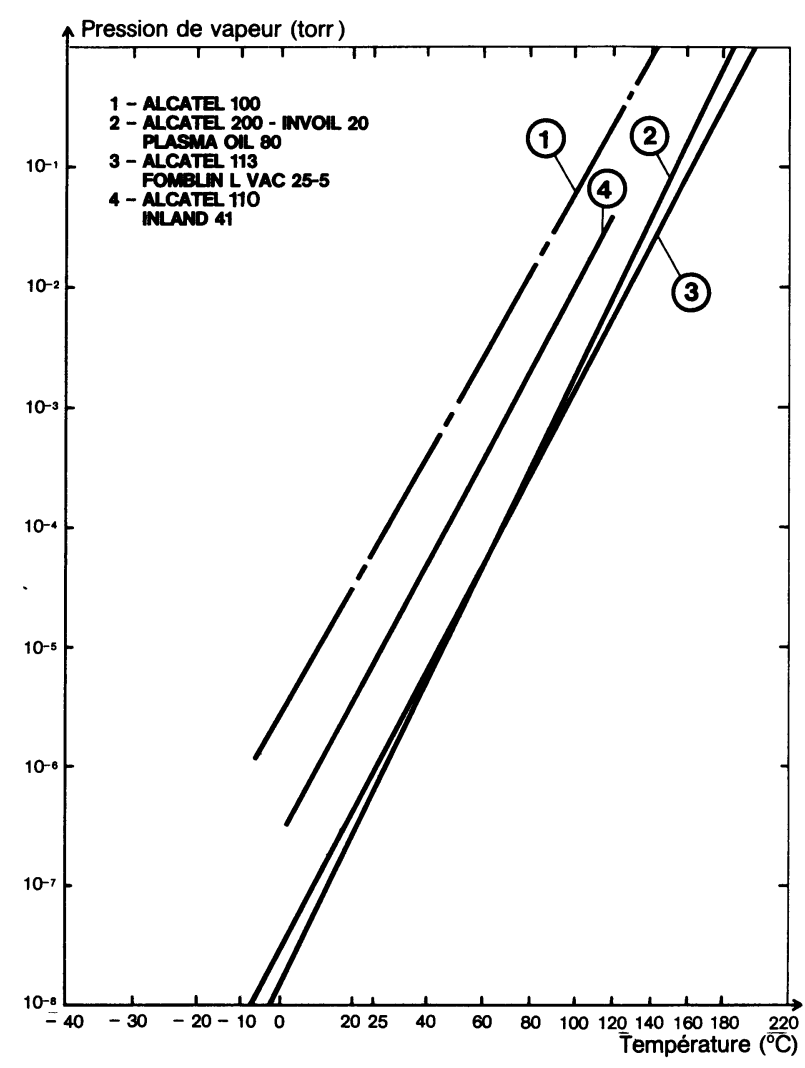

Fig. 2. - Pression de vapeur saturante de quelques huiles pour pompes primaires.

[Vapour pressure of some mechanical pump oils.]

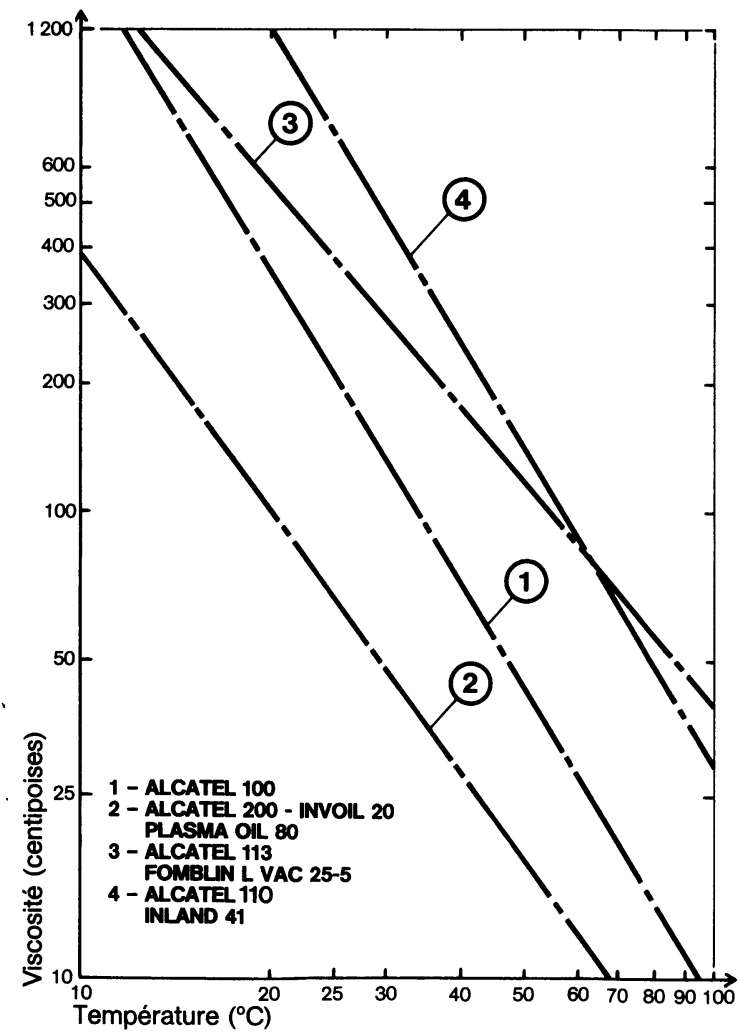

Fig. 3. - Viscosité des huiles pour pompes primaires.

[Mechanical pump oils viscosity vs. temperature.] 
En phase gazeuse dans le réacteur, ce produit se condense sur les points froids de l'installation sous forme d'une poudre de très fine granulométrie (dimensions inférieures au $\mu \mathrm{m}$ ). Les installations sont bien équipées d'un piège pour arrêter ces substances mais le condensat est pulvérulent et est véhiculé par le flux gazeux lors de chaque pompage. Le premier réflexe est d'installer un filtre à poussières sur la canalisation de pompage pour protéger la pompe à vide. Pour être réellement efficace, un tel filtre doit posséder un ensemble de qualités qui le rend cher et encombrant :

- Seuil de rétention très bas $(\leqslant 0,3 \mu \mathrm{m})$.

- Non corrodé par les acides du procédé.

- Conductance suffisante pour ne pas réduire trop sensiblement le débit des pompes.

- Cartouche en matériau ininflammable.

Si cette solution, bien que contraignante sur la maintenance si l'on veut éviter un colmatage important, est envisageable avec des pompes de débits modestes, elle devient pratiquement inemployable avec des groupes de pompage puissants.

Dans les installations où les filtres sont proscrits pour les mêmes raisons que les pièges (sécurité) une solution est d'installer un circuit de pompage lent en prévidage, à l'aide d'une vanne et d'un étranglement.

Il faut savoir que les jeux internes des pompes à vide, même de celles qui sont réputées en avoir de très faibles, sont grands devant les dimensions moyennes des particules de poussières et que l'introduction de corps solides en faibles quantités, ne nuit guère à leur fonctionnement. Les particules passent au- travers de la pompe, sans dommage pour elle. C'est l'accu- mulation de particules dans l'huile qui, par l'intermédiaire du circuit de lubrification, conduit à la dégradation de certains organes des pompes (plus spécialement les paliers et les joints d'arbres tournants). Si donc, on empêche l'accumulation de poussières dans l'huile, en la filtrant, on maintient les performances des pompes sur un temps très long.

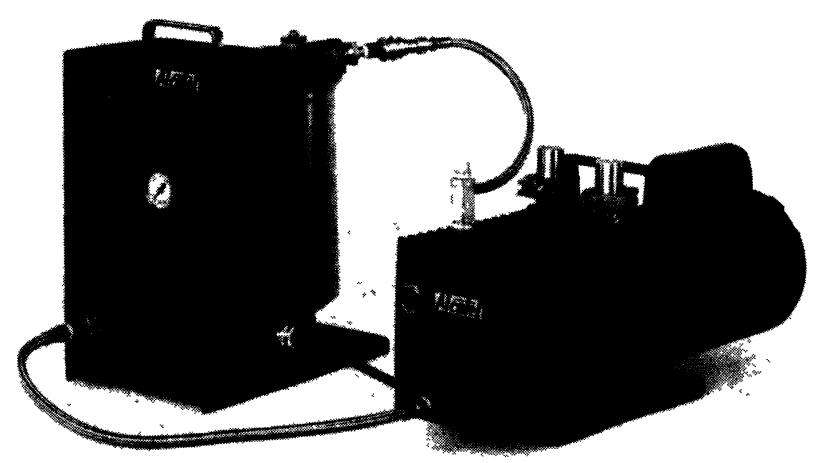

Fig. 4. - Dispositif de filtration d'huile pour système d'implantation ionique.

[Oil filtration system used in ion implant source.]

Le système de filtration d'huile (tel que celui représenté sur la figure 5) a donc plusieurs fonctions :

a) arrêter les corps solides ayant traversé la pompe (chlorure d'ammonium, silice, nitrures,...),

b) retenir les goudrons et les produits de décomposition résultant de l'action des acides sur l'huile,

c) retenir en partie notable les acides en dissolution dans l'huile.

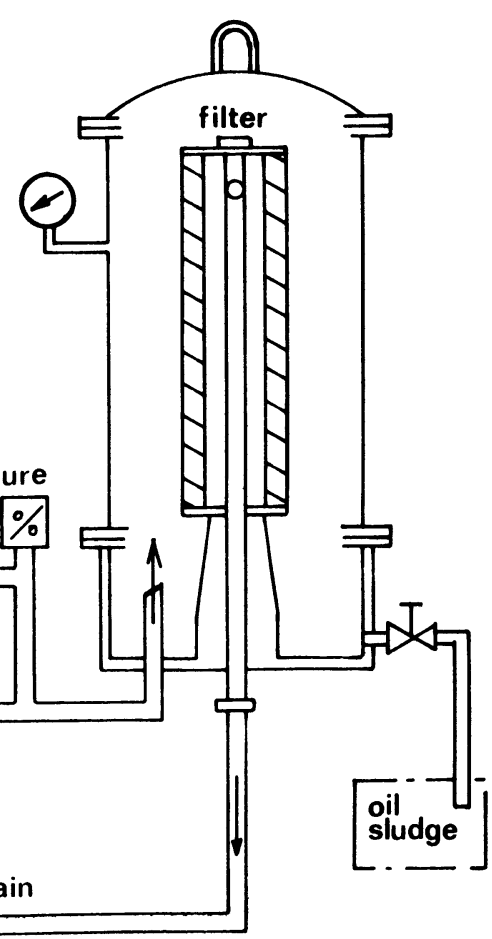

Fig. 5. - Automatic oil filtration system.

[Automatic oil filtration system.] 
Ce dernier phénomène est complexe, mais mesurable, et est la somme des effets provoqués par :

1. L'absorption par les goudrons.

2. L'adsorption physique (physi-sorption) sur la silice et les autres composés solides.

3. L'adsorption chimique (chimi-sorption), ou la fixation par réaction chimique de $\mathrm{HCl}$ sur la cellulose du papier constituant l'élément filtrant.

Avec de tels systèmes (voir photo Fig. 6) les fréquences de vidange de l'huile, et de remplacement des cartouches dépendent beaucoup de la productivité de la machine sur laquelle ils sont montés.
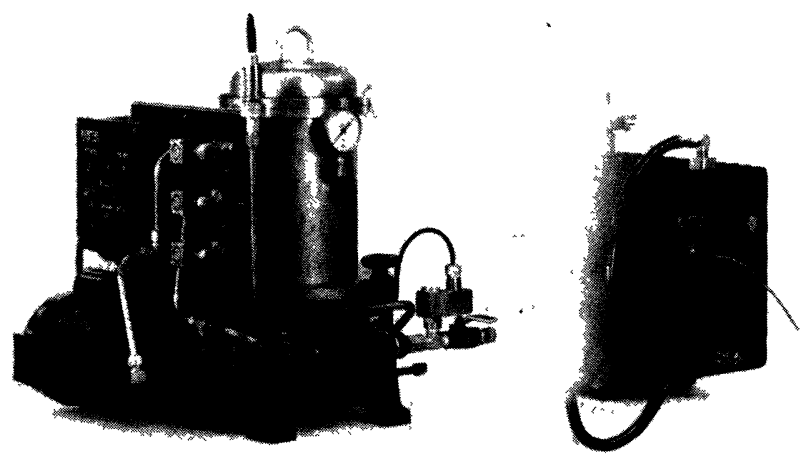

Fig. 6. - Dispositif de filtration d'huile pour L.P.C.V.D.

[Large external oil filtration device for L.P.C.V.D.]

Quelques valeurs sont indiquées dans le chapitre suivant "Maintenance". Les quelques chiffres cidessous donnent une idée assez claire de l'importance des dispositifs de filtration d'huile.

Au bout de $200 \mathrm{~h}$, on recueille dans le filtre environ $6 \mathrm{~kg}$ de boue. Sur un autre système l'expérimentation a été poussée jusqu'à $700 \mathrm{~h}$ de dépôt, avec vidange de l'huile toutes les $100 \mathrm{~h}$. On a recueilli dans le filtre environ $12 \mathrm{~kg}$ de boue. Ces chiffres montrent clairement la nécessité de disposer de filtres de grande surface, pour éviter un colmatage trop rapide. On peut signaler que la perte de charge avec une cartouche filtrante de $3,2 \mathrm{~m}^{2}$ de surface atteint près de 2 bars ( $28 \mathrm{PSi}$ ) au bout de quelques dizaines de dépôts et qu'elle est complètement obturée (Fig. 7).

5. La maintenance. - A notre connaissance, peu de publications abordent ce problème, pourtant si important pour l'utilisateur, probablement parce que les différentes machines disponibles sur le marché ont des comportements assez différents. Nous citerons toutefois le travail de William A. Brown et Théodore I. Kamins [30].

Nous donnerons ci-après quelques exemples, obtenus sur des installations en service, à titre d'illustration. Nous pensons que ces renseignements sont extrapolables.

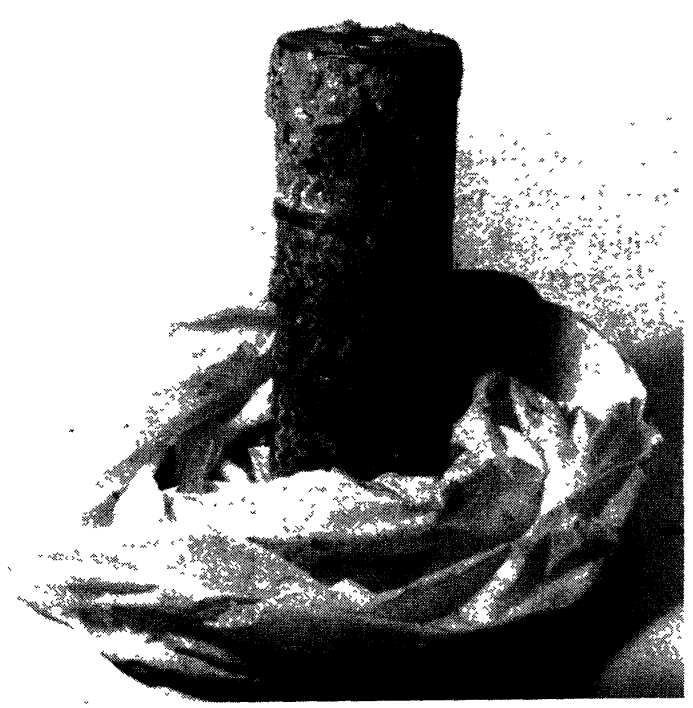

Fig. 7. - Cartouche de filtre à huile colmatée après environ $200 \mathrm{~h}$ de fonctionnement L.P.C.V.D. (dépôt de nitrures).

[Paper filter cartridge clogged after $200 \mathrm{~h}$ of nitride deposition.]

5.1 L.P.C.V.D. - Le problème majeur est celui posé par les systèmes de dépôts de nitrures. Lorsque les pompes qui équipent ces systèmes ne sont pas munies de filtres (cas le plus fréquent), on constate un colmatage rapide du tamis monté dans la canalisation d'aspiration, conduisant souvent à un nettoyage quotidien (après démontage). Deux remèdes sont possibles :

a) installer un piège mécanique (vide de maille $-0,1 \mathrm{~mm})$;

b) monter une injection d'huile auxiliaire par l'aspiration (Fig. 8) pour laver la pompe tous les matins. Cette modification sur un système a permis de démonter le tamis toutes les $800 / 900 \mathrm{~h}$ au lieu d'une fois par semaine. (Installation Tempress en service : Western Electric.)

En dehors de ce problème, on peut résumer les résultats dans le tableau ci-dessous :

Pompe sans filtre

Avec filtre interne petit modèle $\left({ }^{*}\right)$

Avec filtre externe grand modèle

$100 / 200 \mathrm{~h}$

$600 / 700 \mathrm{~h}$

$\left({ }^{*}\right)$ Le filtre interne est une cartouche filtrante, type Automobile montée sur le circuit d'huile de la pompe. L'huile utilisée est de l'huile minérale classique pour pompes à vide (ALCATEL 100-103).

La rentabilité de l'installation d'un tel système est résumée ci-après.

Le calcul du coût mensuel d'exploitation est fait dans l'hypothèse de 200 dépôts par mois (200 h efficaces). 
Pompe SANS filtre externe (petit filtre seulement)

Huile

Cartouches filtrantes

Main-d'œuvre pour :

- Vidange

- Changement de filtre Total

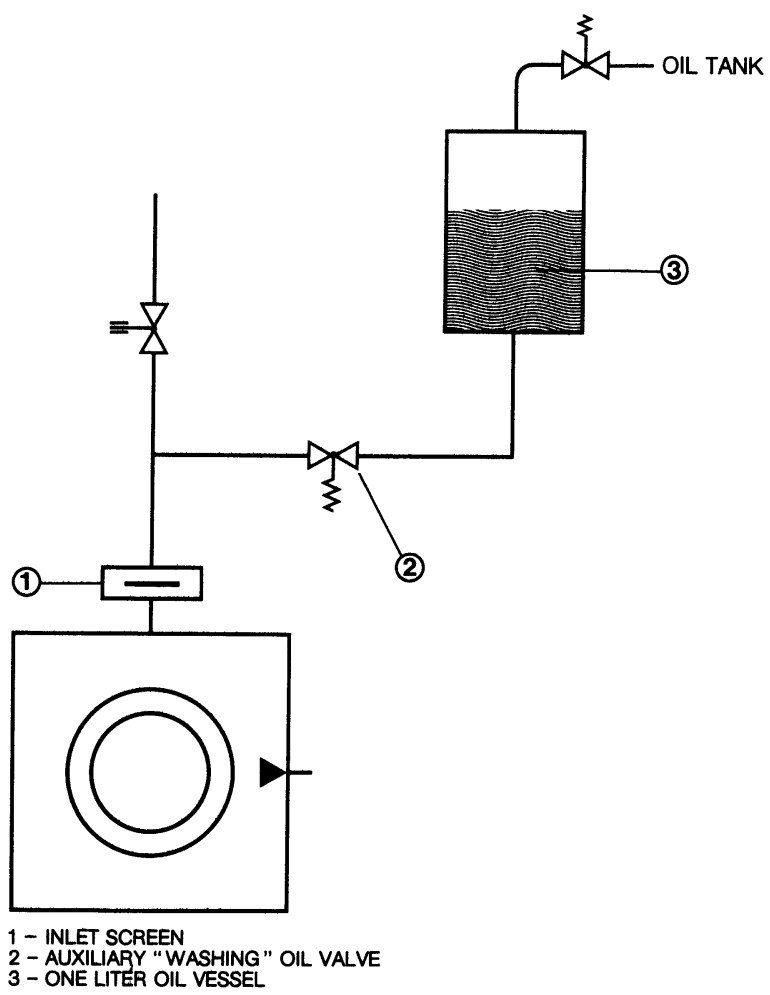

Fig. 8. - Dispositif de lavage par l'aspiration, le remplissage du réservoir d'huile et l'ouverture de la vanne 2 , sont commandés par une minuterie (timer)

[Mechanical pump "inlet oil flushing ". The filling of the oil reservoir and the valves sequency are drived by a timer.]

Dans ces conditions, et sans tenir compte des pertes de productivité entraînées par les arrêts de la machine, on conclut à un amortissement du système auxiliaire en environ 1 trimestre.

5.2 Implantation ionique. - En fait, seule la pompe installée sur la source d'ions travaille réellement dans des conditions difficiles. Les résultats suivants ont été obtenus avec une pompe ALCATEL 2008 AC (Implanteur Extrion installé chez R.C.A.). Il semble que l'utilisation conjointe d'une huile distillée sous vide, à très basse tension de vapeur satu-

\begin{tabular}{lcc}
\multicolumn{1}{c}{ Type d'huile } & $\begin{array}{c}\text { Fréquence } \\
\text { de vidange }\end{array}$ & $\begin{array}{c}\text { Observations } \\
\text { ALCATEL } 103\end{array}$ \\
$8 / \overline{-}$ & $\begin{array}{l}\text { Grosse répara- } \\
\text { tion après } 250 \mathrm{~h} \\
\text { (rebuild) }\end{array}$ \\
$\begin{array}{l}\text { ALCATEL 200 } \\
\begin{array}{l}\text { ALCATEL 200 avec filtre à } \\
\text { huile DE }\end{array}\end{array}$ & $40 \mathrm{~h}$ & \\
& $>300 \mathrm{~h}$ &
\end{tabular}
$80 \mathrm{~h}$. permanence. piège. limite nécessaire; les $40 \mathrm{~h}$
Pompe AVEC filtre externe

$$
\begin{aligned}
301 \times 15 & =450 \\
1 \times 110 & =110 \\
2 \times 20 \mathrm{mn} \times 60 & =40 \\
2 \mathrm{~h} \times 60 & =120 \\
\hline \mathrm{FF} & =720
\end{aligned}
$$

rante, et d'un filtre externe puisse remplacer avantageusement l'utilisation d'huile fluorée. Des pompes en service depuis environ 4 ans ( $8 \mathrm{~h}$ par jour) avec huile Fomblin montrent en effet des traces anormales d'usure au joint d'arbre et aux paliers.

5.3 Attaque sous Plasma. - Les problèmes sont différents selon les procédés et actuellement encore difficiles à appréhender, à l'exception de l'utilisation de l'oxygène en R.I.E. (Reactive Ion Etching) qui tend à imposer l'usage des fluides synthétiques; de ce fait, on dispose d'assez peu de données comparatives. On peut noter avec le mélange $\mathrm{CF}_{4}+\mathrm{O}_{2}$ :

- Huile ALCATEL 103, sans filtre, durée $\simeq 30 \mathrm{~h}$. - Huile ALCATEL 200, sans filtre, durée $\simeq 60$ /

Dans les 2 cas précédents au bout de ce temps, l'huile est devenue très visqueuse.

$\left.\begin{array}{l}\text { - ALCATEL } 110 \\ \text { ou INLAND } 41\end{array}\right\} \begin{aligned} & \text { avec filtre DE : pas de modifi- } \\ & \text { cation après } 6 \text { mois d'utilisa- } \\ & \text { tion. }\end{aligned}$

Il faut noter que l'usage d'huile synthétique sans dispositif de filtration auxiliaire peut conduire, dans certains cas, à une dégradation de la pompe, car les produits corrodants n'étant plus neutralisés par l'huile, s'accumulent dans le bain qui est recyclé en

En ce qui concerne le procédé utilisant $\mathrm{C} \mathrm{Cl}_{4}$ on peut citer l'expérience suivante faite sur machine PLASMA FAB (Electrotech).

Le système de pompage comprend une pompe ALCATEL $2030 \mathrm{C}$, précédée d'un piège à azote liquide. La procédure d'utilisation est la suivante :

- Piège rempli le matin, en même temps que la pompe est mise en marche.

- Pompe arrêtée le soir et isolée par une vanne du

- Piège réchauffé le soir et vidangé par lavage.

Système initial

avec huile ALCATEL 103,

sans dispositif de filtration
Système avec huile chlorocarbonée INLAND 41 et filtre externe DE

- Au bout de 2 jours, on a des Après 8 mois en laboratoire de difficultés à obtenir la pression R.D. (90 h effectives d'attaque) : - Chargement de l'huile toutes - pompe comme neuve, - cartouche filtrante inchangée, obtention instantanée de la pression limite. 


\section{Bibliographie}

[1] Bawa, M. S., Goodmann, R. C. and Truitt, J. K., Proc. 4th Int. CVD Conf., Electro Chem. Soc. (1973).

[2] Baron, M. and Zelez, J., Solid State Technol. 21 (1978) 61

[3] Boyd, H. and TANG, M. S., Solid State Technol. 22 (1979) 133.

[4] Caporiccio, G. and Steenrod, R. A., J. Vac. Sci. Technol. 15 (1978) 775.

[5] Coburn, J. W. and Kay, E., Solid State Technol. 22 (1979) 117.

[6] Coburn, J. W. and Kay, E., J. Vac. Sci. Technol. 16 (1979) 407.

[7] Cochet, G. H., Mellotee, J. P. and Delbourgo, R., J. Electrochem. 125 (1978) 487.

[8] Coburn, J. W. and Winters, H. F., J. Vac. Sci. Technol. 16 (1979) 391.

[9] Duval, P., Vak. Techn. (1979).

[10] Duval, P., Ind. Res. Devel. 21 (1979) 167.

[11] Flamm, D. L., Solid State Technol. 22 (1979) 109.

[12] Kern, W. and Schnable, J. L., I.E.E.E. Trans. Electron Devices 26 (1979) 647.

[13] Gieske, R. J., McMullen, J. J. and Donaghey, Lee F., Proc. 6th Int. CVD Conf., Electro. Chem. Soc. (1977) p. 183.

[14] Hammond, M. L., Solid State Technol. 21 (1978) 68.

[15] Hammond, M. L. and Gieske, R. J., Int. Publication TEMPRESS No 501 (1976).

[16] HiEber, K. and Stolz, M., Siemens Forsch. Entwicklungsber. 6 (1977) 232.
[17] KeRN, W., Solid State Technol. 18 (1975) 25.

[18] Le Claire, R., Solid State Technol. 22 (1979) 139.

[19] Lehmann, W. H. and Widmer, R., J. Vac. Sci. Technol. 15 (1978) 319.

[20] Markstein, H. W., Elect. Pack. Production 17 (1977) 31.

[21] Mauer, J. L., Logan, J. S., Zielinski, L. B. and SchwartZ, C. C., J. Vac. Sci. Technol. 15 (1978) 1734.

[22] Raby, B. A., J. Vac. Sci. Technol. 25 (1978) 205.

[23] Rand, M. J., J. Vac. Sci. Technol. 16 (1979) 420.

[24] Rosler, R. S., Solid State Technol. 20 (1977) 63.

[25] Schaible, P. M., Metzger, W. C. and Anderson, J. P., J. Vac. Sci. Technol. 15 (1978) 334.

[26] Sedwick, T., Proc. 6th Int. CVD Conf., Electro-Chem. Soc. (1977) p. 59.

[27] Brown, H. L., Bunyard, G. B. and Lin, K. C., Solid State Technol. 21 (1978) 35.

[28] Yee, K. K., Proc. Conf. 5th Int. CVD Conf. (1975) p. 283293.

[29] Rosler, R. S. and Engle, G. M., Solid State Technol. 22 (1979) 88.

[30] Brown, W. A. and Kamins, T. I., Solid State Technol. 22 (1979) 51.

[31] Rовв, F., Semi Conductor International (1979) p. 60

[32] Weikel, T. D. and Yven, H. H., Vacuum Pump Explosion Study, U.S. Navy NAEC, GSED 60, code 28638 (1972). 